\title{
Differential Effects of Specific p53 Mutations on Centrosome Structure and Function
}

\author{
Vivian Negron, Catherine Gibson, Kathleen Kitzmann, Wilma Lingle \\ Experimental Pathology, Mayo Clinic \\ 200 First St. SW \\ Rochester, MN 55905
}

Our recent studies of p53 mutations in human breast cancer demonstrated that tumors with mutant p53 tend to have a significantly greater capacity to nucleate microtubules (MTs) than do tumors with wild type p53 [1]. MT nucleation is a major function of the centrosome during both interphase and mitosis. The interphase MT cytoskeleton, through interactions with the actin and intermediate filament cytoskeletons, affects cell polarity, vesicular trafficking, cell-cell adhesions, and cell migration. During mitosis, duplicated centrosomes nucleate the microtubules of the bipolar mitotic spindle apparatus. Centrosome amplification can affect the interphase and mitotic centrosome functions by disrupting cell polarity and by increasing the frequency of multipolar mitoses [2,3]. In breast tumors, the extent of centrosome amplification correlates with the level of chromosomal instability (CIN), supporting the hypothesis that centrosome amplification can cause improper chromosome segregation through the formation of multipolar mitotic spindles [1].

To further our understanding of the relationship between p53 and centrosome function, we have created two adenovirus vectors, M114 and M72, containing two different p53 mutations. We chose the p53 mutations based on the characteristics of the tumors in which they were found. Tumor BT0072, with p53 mutant M72, had 3 fold greater MT nucleating capacity, 10 fold greater centrosome number, and 3.4 fold greater CIN level than normal breast tissues. BT0114, with p53 mutant M114, had 11 fold greater MT nucleation capacity, 3 fold greater centrosome number, and 1.5 fold greater CIN than normal breast tissues. We used the adenoviral vectors to deliver the mutant p53 to cultured human mammary epithelial cells (hMECs) and hMECs previously immortalized with human telomerase (hTERT-hMECs). hMECs, which were derived from normal breast epithelium, senesce after 18 to 22 passages, while hTERT-hMECs grow indefinitely. Infection rates of greater than $90 \%$ were achieved with the adenovirus vectors, and infected cells continued to express mutant protein for more than 2 weeks. We assayed infected cell cultures at intervals after infection to determine if the 2 specific mutant $\mathrm{p} 53 \mathrm{~s}$ induced different alterations in centrosome structure and function. Specifically, we measured frequency of multi- and micro-nuclei, centrosome size, centrosome number, abnormal mitoses, and CIN of chromosomes 3, 7, and 17 and assessed tubulin isoforms and acetylated MT distribution.

Cells infected with either M72 or M114 had higher frequencies of multinucleate cells and cells with amplified centrosomes than did cells infected with a GFP-expressing control virus or uninfected cells (Fig 1, top panel). However, the cells infected with M114 had a much higher frequency of multipolar mitoses than did the cells infected with M72 or control cells (Fig 1, bottom panel). M72 infected cells had a higher frequency of anaphase bridges than M114 infected cells or control cells. By day 4 post infection, CIN increased from 10\% to $15 \%$ in M72 infected cells, but returned to normal levels by day 14 (Fig 2). In sharp contrast, CIN increased to $30 \%$ by day 2 in M114 infected and remained at that level through day 14, the last day tested. Preliminary results indicate that there 
are differences between M72, M114, and control cells in the expression of tubulin isoforms and post-translational modifications of tubulin. These results indicate that specific p53 mutations have different effects on centrosome structure and function and that mutant p53 can affect the microtubule cytoskeleton. These differences likely would have profound in vivo effects on tumor progression and metastasis. Identification of particular p53 mutations in the tumors of breast cancer patients and understanding the specific effects of these mutations on centrosomes, the cytoskeleton, and chromosomal instability may aid in developing custom tailored chemotherapeutic treatments.

\section{References}

[1] Lingle et al., PNAS. 99 (2002) 1978.

[2] Lingle et al., Am. J. Pathol. 155 (1999) 1941.

[3] Lingle et al., PNAS. 95 (1998) 2950.

[4] This work was supported by the Fraternal Order of the Eagles' Cancer Research Award and Department of Defense Breast Cancer Research Program Award DAMD17-98-1-8122 to WL.
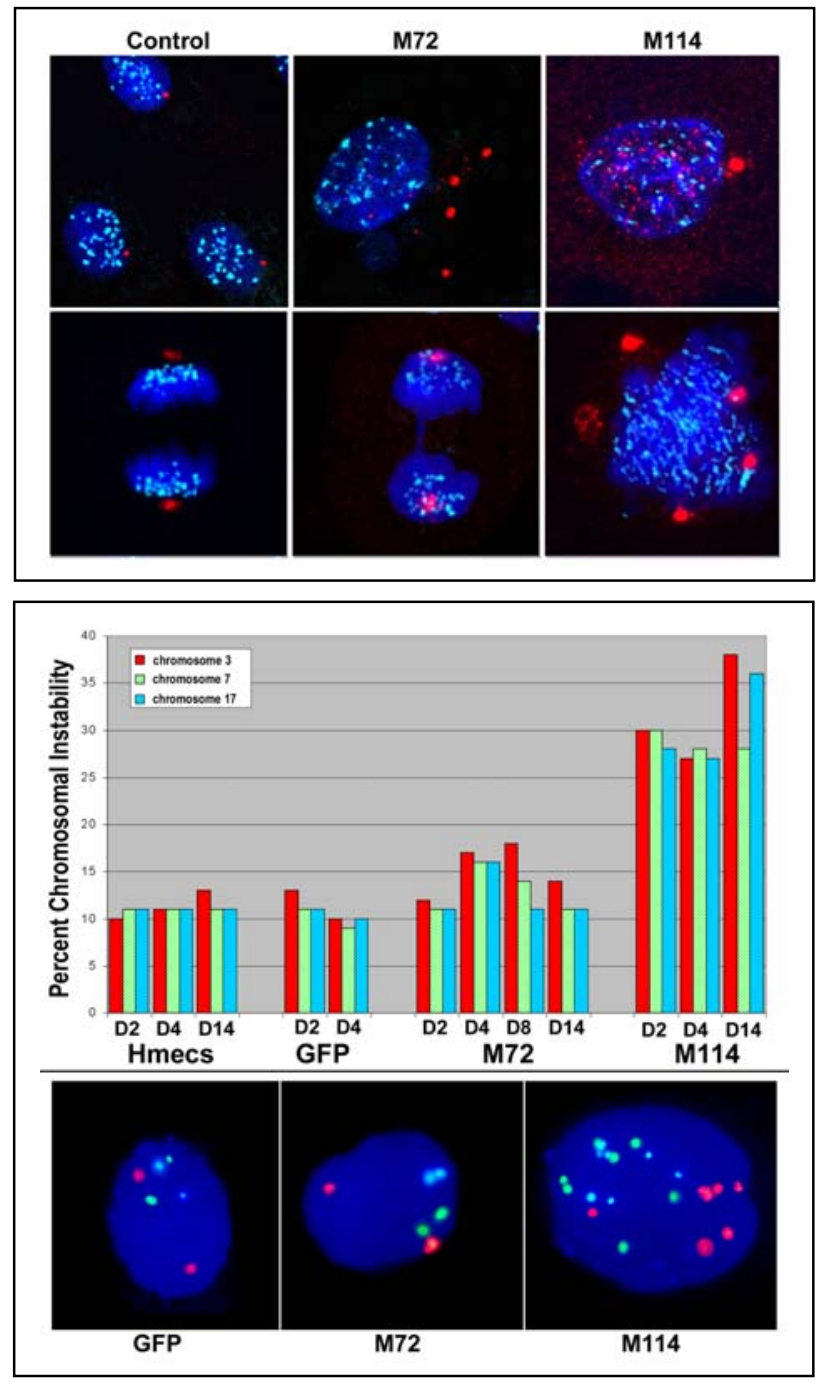

Figure 1. Top panel. On average, control cells have one small centrosome (red) per cell and that centrosome tends to be close to the nucleus (blue). Centrosomes in cells infected with M72 or M114 are larger and/or more numerous and may be farther from the nucleus. Kinetochores are shown in light blue. Bottom Panel. Control cells have few abnormal mitoses. Anaphase bridges are frequent in cells infected with M72, while cells infected with M114 have the highest frequency of multipolar mitoses.

\section{Figure 2. Top Panel. Uninfected and GFP} infected control cells averaged $11 \%$ chromosomal instability. Chromosomal instability was slightly higher in M72 infected cells on days 4 and 8 post infection. Cells expressing M114 mutant p53 had an immediate and sustained increase in chromosomal instability of greater than $25 \%$. Bottom Panel. Numbers in the bar graph were calculated based on triple color FISH using centromeric probes to chromosomes 3 (red), 7 (green), and 17 (blue). 